\title{
Probing the limits of selectivity in a recognition-mediated reaction network embedded within a dynamic covalent library
}

\author{
Tamara Kosikova ${ }^{[a]}$ Harry Mackenzie,${ }^{[a, b]}$ and Douglas Philp ${ }^{\star[a]}$
}

\begin{abstract}
Two recognition-mediated reaction processes operating through a reactive binary complex drive resolution of a 24component dynamic covalent library, assembled from individual aldehydes and nucleophiles. The effectiveness of the library resolution and selective amplification of one recognition-enabled species over another is limited by the difference in the rates of the recognition-mediated reactive processes and strength of the recognition processes employed in the dynamic system.
\end{abstract}

\section{Introduction}

Complexity in the world around us does not simply develop ${ }^{[1]}$ as a function of the increasing number of components within a given system. Instead, it emerges within systems that possess the ability to dynamically self-organise and in which feedback between components is present. The capacity of individual components within a system to interact allows these individual elements, with established properties, to come together and form constructs of higher order, giving rise to emergent behavior $^{[2]}$ and properties. Simultaneously, the interconnectedness of all components enables complex systems to constantly adapt and evolve in response to environmental stimuli. Our inability to control and predict the behaviour of complex systems is an essential feature that allows us to differentiate them from systems that are merely complicated, such as machines or engines, where components interact in a controlled and predictable manner. Only by exploring ${ }^{[3]}$ the role of connectivity and the fundamental relationship between the components of interconnected networks can we begin to develop an understanding of how complex systems may have played a role in the transition from simple compounds to the first self-sustaining system.

The study of a complex chemical network begins with its design. One method that has been shown to be particularly effective in generating complex interconnected systems is dynamic covalent chemistry ${ }^{[4]}$ (DCC). The general combinatorial approach and reversible bond formation allow facile construction of dynamic covalent libraries ${ }^{[5]}$ (DCLs) of interconverting components under thermodynamic control. When a DCL is instructed by the addition of an external stimulus, the equilibrium distribution is altered and the library components stabilised by the interaction with the stimulus are amplified. However, the purely thermodynamic selection limits

[a] T. Kosikova, Prof. D. Philp

School of Chemistry and EaStCHEM

University of St Andrews

North Haugh, St Andrews, KY16 9ST, United Kingdom

E-mail: d.philp@st-andrews.ac.uk

[b] H. Mackenzie

UCB

216 Bath Road, Slough, Berks, SL1 3WE, United Kingdom

Supporting information for this article is given via a link at the end of the document. the magnitude of amplification that can be achieved within the DCL. In order to attain increased selectivity within DCLs, we have to employ methods that couple DCLs to irreversible processes and allow the target to be removed from a pool of exchanging components through kinetic selection. In the past, several successful examples, demonstrating target amplification using both chemical reactions ${ }^{[6]}$ and physical processes $^{[7]}$, have been reported. Recently, we have reported $^{[8]}$ the use of a single, recognition-mediated irreversible 1,3-dipolar cycloaddition reaction as the means of selecting and amplifying a specific target from within a dynamic library composed of interconverting nitrones and imines. Specifically, we explored two different kinetic modes for achieving DCL resolution (Figure 1), driven by (i) formation ${ }^{[8 b]}$ of a reactive binary complex (AB, Figure 1) or (ii) template-mediated self-replication ${ }^{[8]}(\mathrm{SR}$, Figure 1$)$. In this work, we examine the factors that govern the selectivity in a $\mathrm{DCL}$, assembled from individual aldehydes and nucleophiles, incorporating a reaction network involving not one, but two recognition-mediated processes operating through a reactive binary complex. With specific focus on this mode of selection within the $\mathrm{DCL}$, we demonstrate how changing the strength of recognition processes within the system influences the effectiveness of resolution within the library.

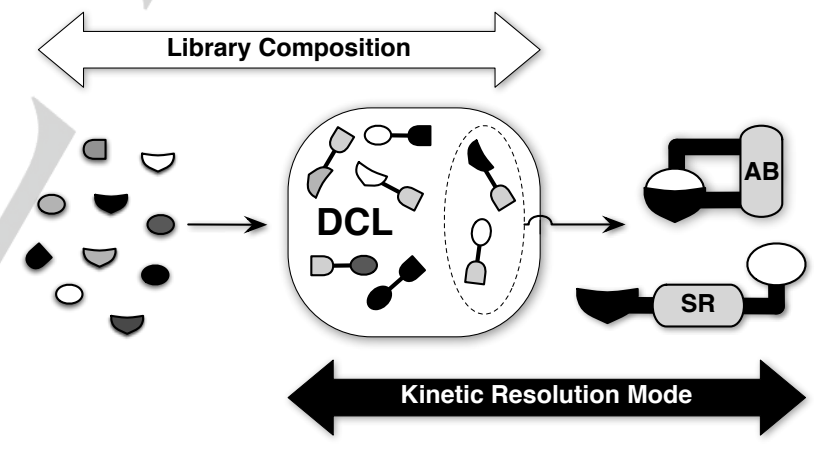

Figure 1. Resolution of a dynamic covalent library ( $D C L)$ depends on the composition of the library and the mode of resolution employed. AB denotes a resolution mode driven by reaction of library members in a recognitionmediated reaction operating through a binary reactive complex. SR denotes a resolution mode driven by reaction of library members in a recognitionmediated autocatalytic reaction controlled by a self-complementary template.

\section{Results and Discussion}

Our dynamic covalent library (Figure 2) is based on a $4 \times 4$ matrix of aldehydes, $\mathbf{A}$ to $\mathbf{D}$, and nucleophiles, $\mathbf{W}$ to $\mathbf{Z}$, comprised of three amines and one hydroxylamine, which afford an exchange pool of 12 imines and 4 nitrones. The total of 24 interconverting components represents a dynamic system that can be analysed readily by ${ }^{19} \mathrm{~F}$ NMR spectroscopy - the presence of either an aryl-F or trifluoromethyl $\left(-\mathrm{CF}_{3}\right)$ tag on each nucleophilic component facilitating the identification of individual components in the library. 


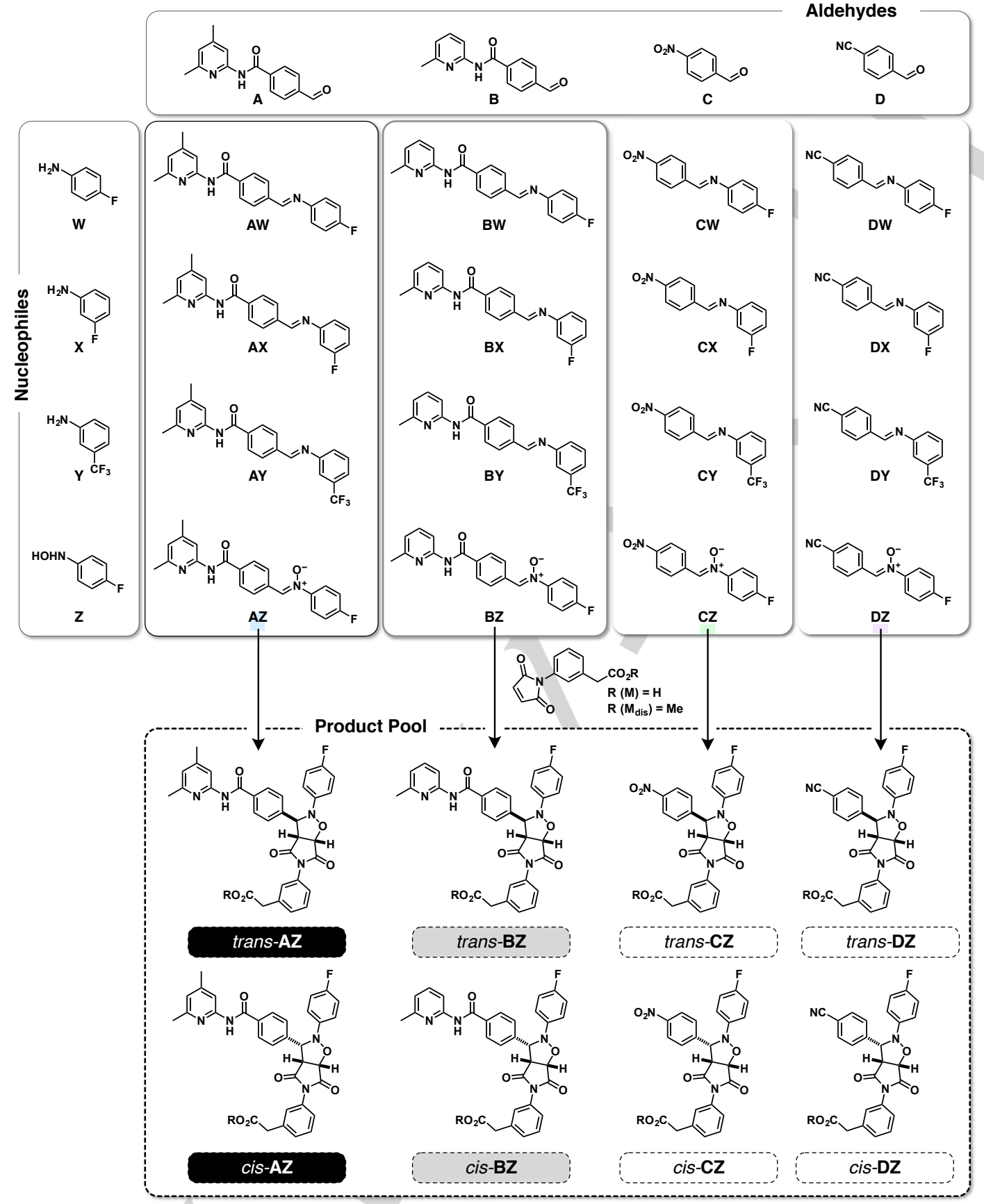

Figure 2. Dynamic covalent library composed of four aldehydes, $\mathbf{A}$ to $\mathbf{D}$ and four nucleophiles, $\mathbf{W}$ to $\mathbf{Z}$. Corresponding exchange pool components and cycloadducts, formed upon reaction with recognition-enabled maleimide $\mathbf{M}$ and recognition-disabled $\mathbf{M}_{\text {dis }}$, containing aldehyde $\mathbf{A}$ are in black, aldehyde $\mathbf{B}$ are in grey, aldehyde $\mathbf{C}$ and aldehyde $\mathbf{D}$ are white.

The library is designed to contain components equipped with two distinct features: a reactive site and a recognition element. Formation of a reactive nitrone is provided by reaction of aldehydes with hydroxylamine $\mathbf{Z}$. The library also contains two aldehydes bearing a site capable of recognising a carboxylic acid: A (4,6-dimethyl amidopyridine) and B (6-methyl amidopyridine). Overall, the library exchange pool contains only two components, $\mathbf{A Z}$ and $\mathbf{B} \mathbf{Z}^{[8]}$, equipped with both a reactive and a 


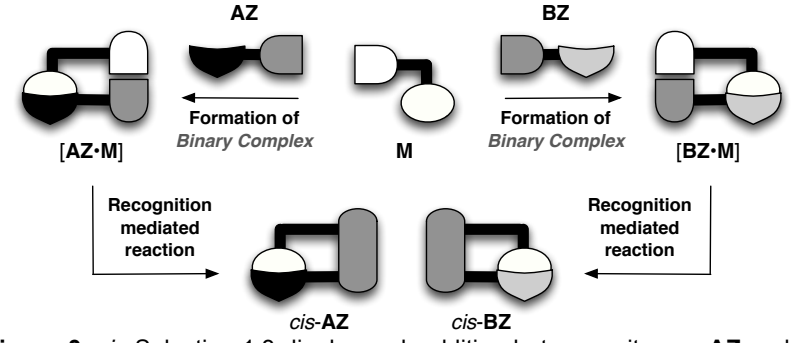

Figure 3. cis Selective 1,3-dipolar cycloaddition between nitrones $\mathbf{A Z}$ and $\mathbf{B Z}$ with maleimide $\mathbf{M}$ proceeds via the recognition-mediated formation of $[\mathbf{A Z} \cdot \mathbf{M}]$ and $[\mathbf{B} \cdot \mathbf{M}]$ reactive binary complexes.

recognition element, required for a recognition-mediated irreversible 1,3-dipolar cycloaddition reaction with a maleimide ${ }^{[8 b]}$ (M). The reactions proceed via formation of the $[\mathbf{A Z} \cdot \mathbf{M}]$ or $[\mathbf{B Z} \cdot \mathbf{M}]$ binary complexes (Figure 3 ) afforded by the recognition of the carboxylic acid bearing maleimide by the amidopyridine recognition site, thus facilitating selective formation of cis cycloadducts.

In order for selective amplification of recognition-enabled species from within a DCL to take place, the irreversible cycloaddition must accomplish the formation of cis-AZ and cisBZ fuelled only by the building blocks distributed amongst the pool of simple components. Whilst both $\mathbf{A Z}$ and $\mathbf{B Z}$ are capable of molecular recognition with a carboxylic acid, the strength of their association constants are expected to be different as a result of the changes in the electronic environment of the pyridine moieties. The pyridine unit on $\mathbf{A Z}$ contains an additional methyl group, increasing the electron density on the pyridine ring, and therefore, the strength of the association with the maleimide $-\mathrm{COOH}$. We estimated the association constants for the formation of $[\mathbf{A Z} \cdot \mathbf{M}]$ and $[\mathbf{B Z} \cdot \mathbf{M}]$ binary complexes, based on a $K_{a}$ measured for a structurally similar phenylacetic acid, to be 315 and $160 \mathrm{M}^{-1}$, respectively (See Supporting Information). The two remaining nitrones, $\mathbf{C Z}$ and $\mathbf{D Z}$, which do not contain a recognition element, can also react with maleimide $\mathbf{M}$, however only via the slow and unselective bimolecular reaction, affording both the trans and cis diastereoisomeric products, typically in a $3: 1$ ratio.

\section{The Exchange Pool}

Initially, we needed to confirm that our library forms a dynamic exchange pool. Determination of the equilibrium distribution of our DCL in the absence of any recognition-mediated reactions will enable us to quantify the magnitude of perturbation of the DCL composition upon addition of instructing maleimide. In a typical experiment, an equimolar solution of aldehydes and nucleophiles $([\mathbf{A}]$ to $[\mathbf{D}]$ and $[\mathbf{W}]$ to $[\mathbf{Z}]=20 \mathrm{mM}$ ) was prepared in $\mathrm{CD}_{2} \mathrm{Cl}_{2}$ saturated with para-toluenesulfonic acid monohydrate (PTSA). The solution was incubated at $273 \mathrm{~K}$ and the product distribution was determined by quantitative ${ }^{19} \mathrm{~F}\left\{{ }^{1} \mathrm{H}\right\} \quad \mathrm{NMR}$ spectroscopy $(282.4 \mathrm{MHz}$ ) after 5 days (for details, see Supporting Information). However, as a result of insensitivity of the $-\mathrm{CF}_{3}$ group to changes in its surrounding electronic environment, we were unable to resolve individual resonances for the $\mathbf{Y}$-imines bearing the trifluoromethyl tag. The distribution of condensation products in the library exchange pool is presented in Figure 4. The equilibrium position for the formation of nitrones from hydroxylamine $\mathbf{Z}$ lies far towards the product side, with almost complete conversion (>99\%) to the corresponding nitrones. The two recognition-enabled nitrones, $\mathbf{A Z}$ and $\mathbf{B Z}$, are present at a higher concentration than nonrecognition nitrones $\mathbf{C Z}$ and $\mathbf{D Z}$. Interestingly, the more electron rich pyridine ring present in aldehyde $\mathbf{A}$ makes it less reactive towards nucleophiles than aldehyde $\mathbf{B}$, resulting in a slightly higher concentration of $\mathbf{B Z}(6.72 \mathrm{mM})$ than $\mathbf{A Z}(6.39 \mathrm{mM})$.

(a)

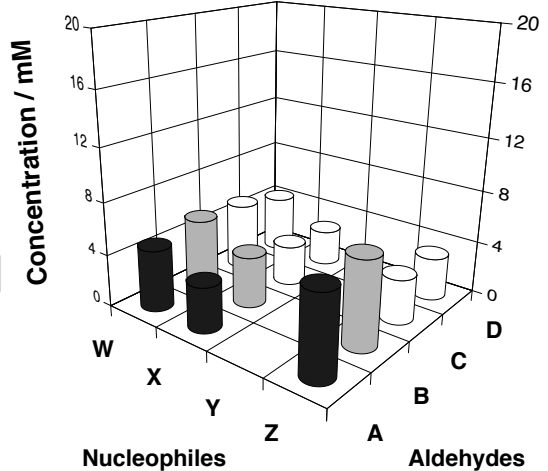

(b)

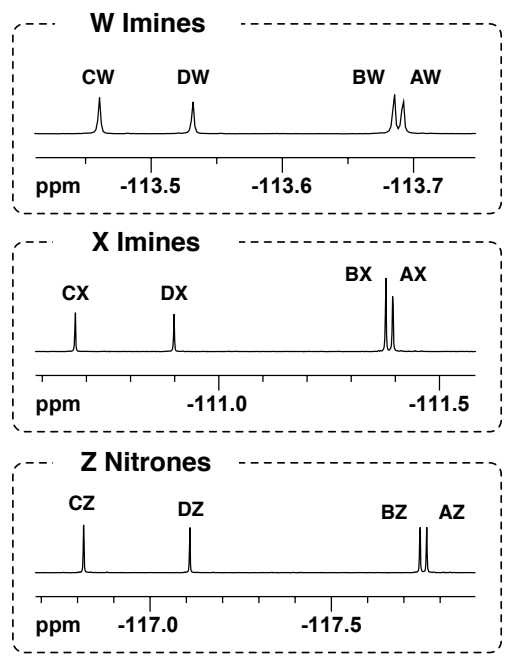

Figure 4. (a) Distribution of the $D C L$ exchange pool after five days at $273 \mathrm{~K}$, as determined by (b) ${ }^{19} \mathrm{~F}\left\{{ }^{1} \mathrm{H}\right\}$ NMR spectroscopy $(282.4 \mathrm{MHz})$, relative to 1-fluoro-4-nitrobenzne as the internal standard $([\mathbf{A}]$ to $[\mathbf{D}]$ and $[\mathbf{W}]$ to $[\mathbf{Z}]=$ $20 \mathrm{mM}$ in $\mathrm{CD}_{2} \mathrm{Cl}_{2}$ saturated with para-toluenesulfonic acid monohydrate). The resonances for $\mathrm{Y}$-imines could not be resolved as a result of the insensitivity of the $-\mathrm{CF}_{3}$ tag to changes in its electronic environment.

\section{Kinetic Selection in the Absence of the DCL}

Prior to instructing the DCL through addition of maleimide $\mathbf{M}$, we needed to rule out inherently different reactivities of the two nitrones towards maleimides as a potential factor contributing to the selectivity for the formation of any one cycloadduct. In this respect, we constructed a series of kinetic experiments (Figure 5) that would establish the reactivity of both $\mathbf{A Z}$ and $\mathbf{B Z}$ nitrones in the absence of any dynamic components. Firstly, the 
individual reactions of each nitrone with a recognition-disabled maleimide $\mathbf{M}_{\text {dis }}$ were examined. The carboxylic acid on $\mathbf{M}_{\text {dis }}$ is protected as the methyl ester, rendering it incapable of recognition-mediated interactions and by examining its reaction with the two recognition nitrones, we can establish the rate constants and diastereoselectivity associated with the bimolecular pathways.

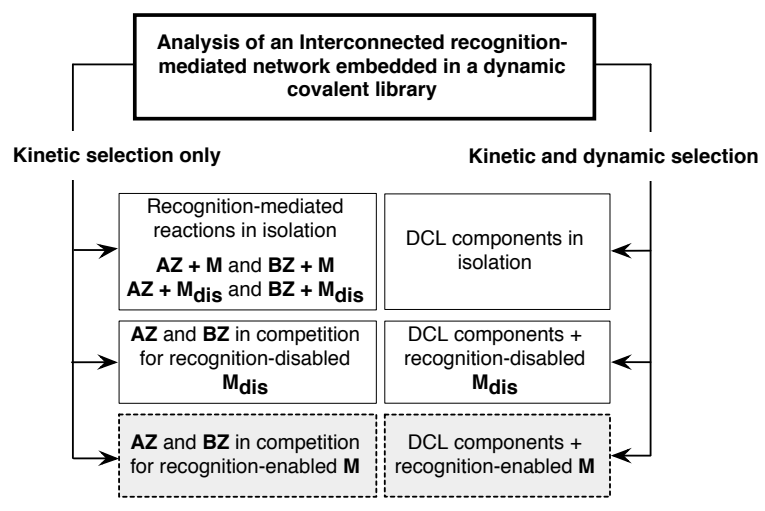

Figure 5. Flow chart outlining a series of experiments designed to probe the origins of selectivity in a recognition-mediated network embedded in a dynamic covalent library. Both the $\mathrm{DCL}$ and the irreversible reactions have to be examined in isolation and in a competition scenario in the presence of recognition-disabled maleimide $\mathbf{M}_{\text {dis }}$ and recognition-enabled maleimide $\mathbf{M}$ (grey box)

Secondly, analysis of the reaction of $\mathbf{A Z}$ and $\mathbf{B Z}$ with a recognition-enabled maleimide $\mathbf{M}$ allows us to determine the rate and selectivity enhancement gained through recognition in a purely kinetic scenario. Thirdly, probing the reactivity of both nitrones under competition conditions, in the presence of either recognition-disabled maleimide $\mathbf{M}_{\text {dis }}$ or recognition-enabled maleimide $\mathbf{M}$ provides us with a direct measurement of selectivity afforded through kinetic selection only. These three isolated kinetic experiments will, ultimately, allow us to evaluate the extent of contribution of dynamic selection to the overall selectivity in the recognition-network. We employed three experiments in order to probe the selectivity within a DCL. Analysis of the exchange pool distribution in the absence of any maleimide was described in Figure 4. Two additional DCL experiments were designed to complement the competition kinetics performed with $\mathbf{M}_{\text {dis }}$ and $\mathbf{M}$ maleimides in the absence of $\mathrm{DCL}$, and are presented in a later section.

In each kinetic experiment, a sample containing the nitrone(s) and the maleimide at $20 \mathrm{mM}$ was prepared in $\mathrm{CD}_{2} \mathrm{Cl}_{2}$ saturated with PTSA and the product evolution was monitored at regular intervals by ${ }^{1} \mathrm{H}$ NMR $(500.1 \mathrm{MHz})$ or ${ }^{19} \mathrm{~F}\left\{{ }^{1} \mathrm{H}\right\} \quad \mathrm{NMR}$ (470.3 MHz) spectroscopy at $273 \mathrm{~K}$ (for details, see Supporting
Information). Kinetic experiments with recognition-disabled maleimide $\mathbf{M}_{\text {dis }}$ confirmed that, in the absence of recognition, the cycloaddition reactions are slow and unselective, resulting in less than $20 \%$ conversion to cycloadducts after 15 hours, with cis:trans diastereoisomeric ratio of $\sim 1: 2.3$ in each experiment. The outcome of analogous reactions with maleimide $\mathbf{M}$ clearly demonstrated the effect of enabling molecular recognition, revealing conversion to cycloadducts of $>85 \%$ after 15 hours and more than $40: 1$ selectivity for the cis diastereoisomer. Concentration-time data for the four isolated kinetic experiments were fitted to the appropriate kinetic model (see Supporting Information), constructed to encompass all the interactions in each system as well as the estimated association constants, thus allowing us to extract kinetic parameters for the individual reactions of $\mathbf{M}$ and $\mathbf{M}_{\text {dis }}$ with nitrones $\mathbf{A Z}$ or $\mathbf{B Z}$ (Table 1).

Table 1. Kinetic and association constants determined for the reaction of $\mathbf{A Z}$ and $\mathbf{B Z}$ with $\mathbf{M}$ and $\mathbf{M}_{\text {dis }}$ by kinetic simulation and fitting of experimental data using the SimFit program.

\begin{tabular}{|c|c|c|c|c|}
\hline & trans-AZ & cis-AZ & trans-BZ & cis-BZ \\
\hline Bimolecular rate constant $/ \mathrm{M}^{-1} \mathrm{~s}^{-1}$ & $1.24 \times 10^{-4}$ & $5.57 \times 10^{-5}$ & $1.30 \times 10^{-4}$ & $5.93 \times 10^{-5}$ \\
\hline Recognition-mediated rate constant $/ \mathrm{s}^{-1}$ & - & $1.24 \times 10^{-4}$ & - & $1.18 \times 10^{-4}$ \\
\hline Effective molarity / $\mathrm{M}$ & - & 2.23 & - & 1.99 \\
\hline Association constant $K_{\mathrm{a}} / \mathrm{M}^{-1}$ & \multicolumn{2}{|c|}{315} & \multicolumn{2}{|c|}{160} \\
\hline
\end{tabular}

Employing the fitting procedure (see Supporting Information), we were able to determine the bimolecular and recognitionmediated rate constants, as well as the respective effective molarities (EMs) associated with $\mathbf{A Z}$ and $\mathbf{B Z}$ nitrones. Nevertheless, when these extracted parameters were employed in a kinetic model simulating the competition reaction between $\mathbf{A Z}$ and $\mathbf{B Z}$ with maleimide $\mathbf{M}$, we observed a disagreement between the estimated $K_{\mathrm{a}}$ values used in the fitting of individual kinetics and the $K_{a}$ values required for achieving the best fit between our model and the competition kinetic data. Such difference suggests that system-level interactions, arising from the transfer of the individual recognition-mediated processes to an environment enforcing competition for a shared building block, exerts an unforeseen effect on the apparent $K_{\mathrm{a}}$ values for the two recognition-processes. Namely, the fitted association constants, specific to the aldehydes and maleimide employed in our system under competition conditions, were determined as 220 and $170 \mathrm{M}^{-1}$ for $[\mathbf{A Z} \cdot \mathbf{M}]$ and $[\mathbf{B Z} \cdot \mathbf{M}]$, respectively (ratio of 1.3:1).

The difference in relative efficiencies of the two recognition processes manifested itself during the competition for a limited supply of maleimide M (Figure 6a). Both recognition-enabled nitrones, $\mathbf{A Z}$ and $\mathbf{B Z}$, exhibited similar reaction efficiency when instructed with $M$, reaching $52 \%$ and $44 \%$ conversion to recognition-enabled products cis-AZ and cis-BZ (ratio of 1.2:1) after 15 hours, respectively (Figure 6b). 


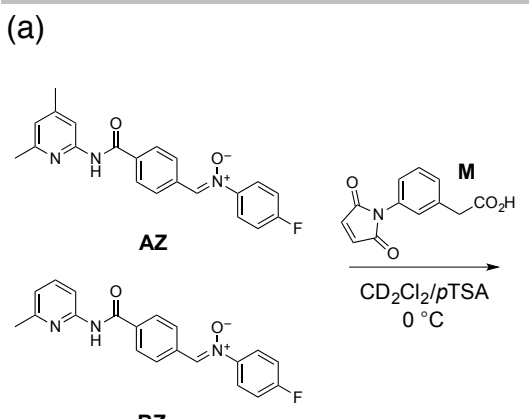

BZ
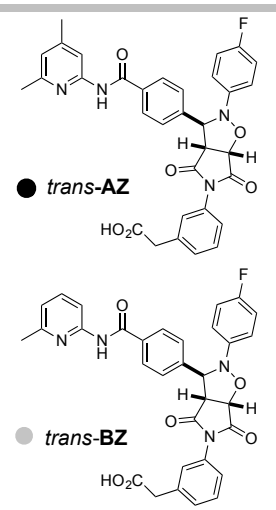

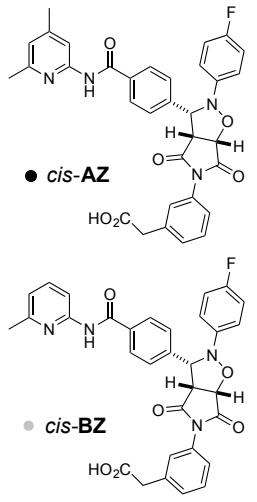

(b)

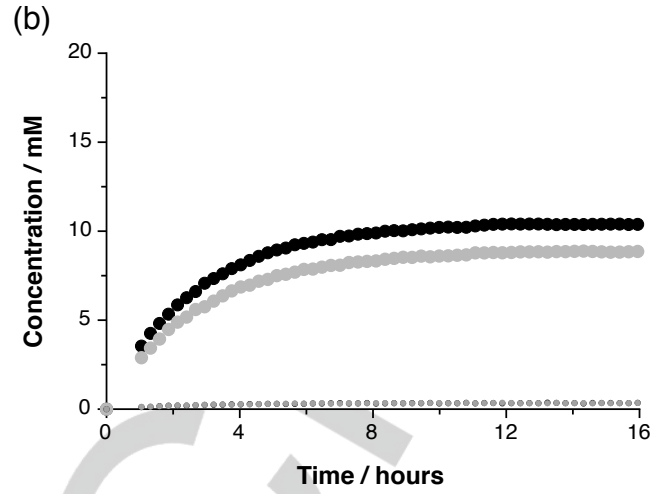

Figure 6. (a) The 1,3 dipolar cycloaddition between nitrone $\mathbf{A Z}$ and $\mathbf{B Z}$ and recognition-enabled maleimide $\mathbf{M}$ gives rise to sets of two diastereoisomeric products: (i) trans-AZ and cis-AZ and (ii) trans- $\mathbf{B Z}$ and cis- $\mathbf{B Z}$. (b) Concentration vs time plot for this reaction $\left([\mathbf{A Z}]=[\mathbf{B Z}]=[\mathbf{M}]=20 \mathrm{mM}, \mathbf{C D}{ }_{2} \mathrm{Cl}{ }_{2}\right.$ saturated with paratoluenesulfonic acid monohydrate, $0^{\circ} \mathrm{C}$ ) as determined by ${ }^{19} \mathrm{~F}\left\{{ }^{1} \mathrm{H}\right\}$ NMR $(470.3 \mathrm{MHz})$ spectroscopy. The data points for the formation of trans-BZ closely mirror the data for trans-AZ, making it difficult to observe the trans-BZ data points on the plot.

Fitting of the concentration vs time data revealed that the enhancement in the recognition mediated-reaction relative to bimolecular rate constant (effective molarity, EM) is largely similar for both reactions, with an EM ratio of 1.1:1 for $\mathbf{A Z : B Z . ~}$ Therefore, the uneven population of cis-AZ and cis- $\mathbf{B Z}$ is predominantly influenced by the different affinities of $\mathbf{A Z}$ and $\mathbf{B Z}$ for $\mathbf{M}$, with smaller contribution exerted also by the individual variations in reactivity. We envisaged that competition for a shared building block might present a different outcome when the reaction network is embedded in a dynamic library. More specifically, we hoped that the absence of preformed nitrones would allow the added maleimide to selectively pull material from the exchange pool and form the product with a higher association constant preferentially, amplifying the difference between the two cis products in the processes.

\section{Recognition-mediated Resolution}

In order to ascertain that simply performing the cycloaddition reaction in the presence of a DCL does not generate any significant changes in composition, the DCL was first addressed with a recognition-disabled maleimide $\mathbf{M}_{\text {dis. }}$. Under recognitiondisabled conditions, all four nitrones can only react through a slow, bimolecular reaction. A library sample ([A] to [D] and [W] to $[\mathbf{Z}]=\left[\mathbf{M}_{\text {dis }}\right]=20 \mathrm{mM}, \mathrm{CD}_{2} \mathrm{Cl}_{2}$ saturated with PTSA) was analysed by ${ }^{19} \mathrm{~F}\left\{{ }^{1} \mathrm{H}\right\}$ NMR spectroscopy $(282.4 \mathrm{MHz})$ after 5 days at $273 \mathrm{~K}$ (Figure $7 \mathrm{a}$ ). The exchange pool distribution remained largely unaffected by the slow and unselective cycloaddition reactions, with only $40 \%$ overall conversion to all 8 cycloadducts. The four nitrones exhibited similar reactivities, and it was clear that simply incorporating an irreversible reaction into a DCL did not afford selectivity for the recognition species. (a)

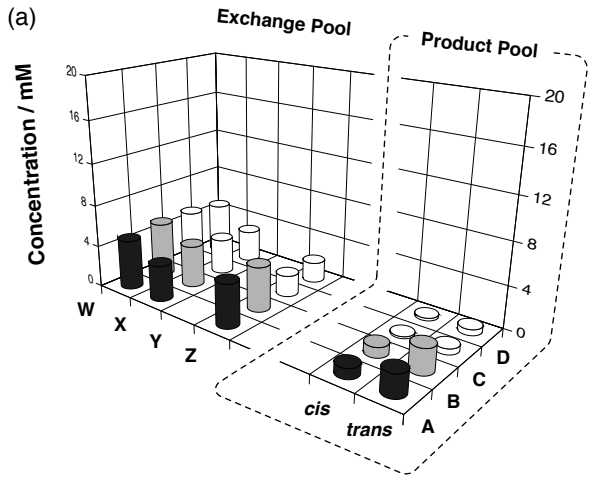

(b)

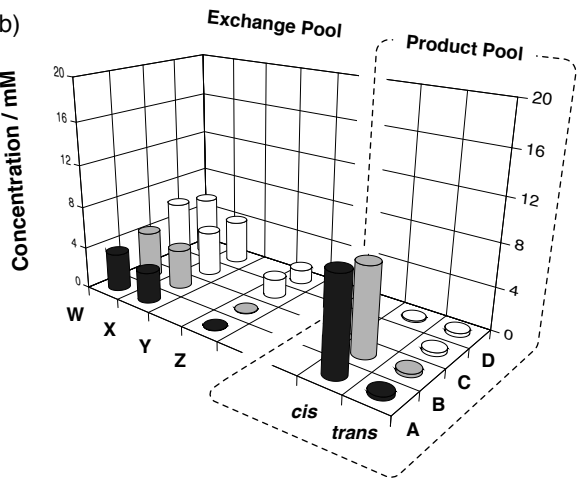

(c)

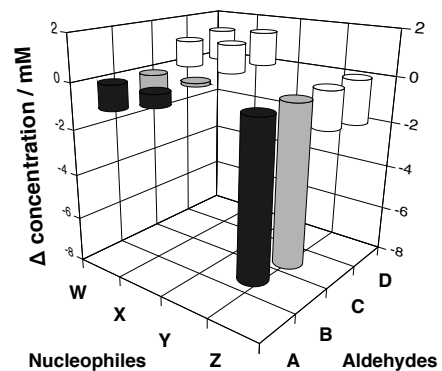

Figure 7. The DCL was instructed with (a) recognition-disabled maleimide $\mathbf{M}_{\text {dis }}$ and (b) recognition-enabled maleimide $\mathbf{M}$. Distribution of the libraries was examined by ${ }^{19} \mathrm{~F}\left\{{ }^{1} \mathrm{H}\right\}$ NMR spectroscopy $(282.4 \mathrm{MHz})$ after five days at $273 \mathrm{~K}$, relative to 1-fluoro-4-nitrobenzne as the internal standard ([A] to [D] and [W] to [Z] and $[\mathbf{M}]$ or $\left[\mathbf{M}_{\text {dis }}\right]=20 \mathrm{mM}$ in $\mathrm{CD}_{2} \mathrm{Cl}_{2}$ saturated with para-toluenesulfonic acid monohydrate). Resonances for $\mathbf{Y}$-imines (in all experiments) and for cis-CZ (b) could not be unambiguously assigned and their concentrations were not determined. (c) Shows the change in exchange pool concentration in a DCL instructed with $\mathbf{M}$ relative to the uninstructed exchange pool experiment after 5 days

We next examined the effect of recognition-enabled maleimide $\mathbf{M}$ on the library distribution and product selectivity. An equimolar solution containing all reaction components ([A] to [D] and $[\mathbf{W}]$ to $[\mathbf{Z}]=[\mathbf{M}]=20 \mathrm{mM}$ ) was prepared in $\mathrm{CD}_{2} \mathrm{Cl}_{2}$ saturated with PTSA and the solution examined by ${ }^{19} \mathrm{~F}\left\{{ }^{1} \mathrm{H}\right\} \quad \mathrm{NMR}$ spectroscopy $(282.4 \mathrm{MHz})$ after 5 days at $273 \mathrm{~K}$. Addition of the recognition-enabled maleimide $\mathbf{M}$ had a profound effect on the distribution of the product pool (Figure $7 \mathrm{~b}$ ). Recognition-enabled cycloadducts cis-AZ and cis- $\mathbf{B Z}$ are present in a ratio of 1.1:1, 
at a significantly higher concentration than any other product, with $45 \%$ and $41 \%$ conversion respectively.

The higher $K_{\mathrm{a}}$ value for the recognition processes in cisAZ manifested itself in the marginally higher proportion of this product than that of cis-BZ. The effect of introducing recognition was also immediately noticeable within the exchange pool. The library components equipped with the recognition site are significantly depleted in response to the decreasing amounts of $\mathbf{A Z}$ and $\mathbf{B Z}$ nitrones. The release of the $\mathbf{W}$ and $\mathbf{X}$ exchange pool components from their $\mathbf{A}$ - and $\mathbf{B}$-imines results in increased concentrations of the $\mathbf{C}$ - and $\mathbf{D}$-imines, incorporating these components. Figure 7c shows the magnitude of the recognition effect on the DCL distribution, highlighting the difference between the concentrations of exchange pool in the recognitionenabled scenario relative to exchange in the absence of any maleimide. The increased concentration of non-recognition imines illustrates how the connectivity of a network can allow system-wide effects to emerge.

\section{Origins of Limited Selectivity}

Selectivity in a system with two simultaneous and irreversible kinetically-driven processes is inherently limited by the reaction conditions and the kinetic parameters governing these processes. The isolated kinetic experiment, where $\mathbf{A Z}$ and $\mathbf{B Z}$ nitrones competed for a limited amount of recognition maleimide $\mathbf{M}$, revealed that the more strongly binding amidopyridine unit allows $\mathbf{A Z}$ to associate and react to a greater extent than $\mathbf{B Z}$ nitrone. The final ratio in this experiment was $\sim 1.2: 1$ in favour of cis-AZ. We hoped that the ratio would be amplified upon transferring the competing recognition-mediated reaction network to a dynamic environment. However, embedding the network within a DCL showed a negligible effect on the formation of these two products and selectivity remained essentially unchanged (Figure 8).
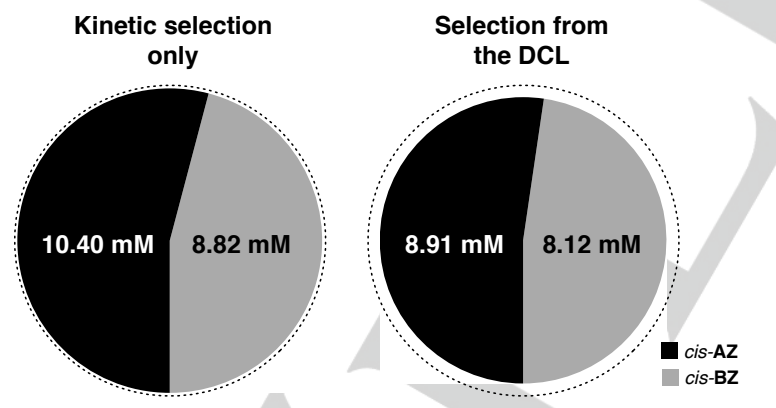

Figure 8. Concentration of cis-AZ (black) and cis-BZ (grey) formed under kinetic conditions after 15 hours (cis-AZ:cis-BZ $=1.2: 1$ ) and within the environment of $D C L$ after five days (cis-AZ:cis-BZ = 1.1:1). The area of the circle denoted by the dotted line represents $100 \%$ conversion of maleimide $\mathbf{M}$, whereas the relative areas of the pie charts are scaled to represent the actual conversion to recognition-enabled products under kinetic selection $(96 \%)$ and kinetic and dynamic selection (85\%).

While the dynamic selection did not provide the desired increase in selectivity, the recognition events exerted a dramatic effect on the product distribution (Figure 7b vs Figure 7a) in the library and facilitated selection of species equipped with recognition elements effectively. AZ, BZ, A, B and $\mathbf{Z}$ exchange pool components were removed selectively from the mixture of interconnected components despite the fact that they were present as minor components. Employing dynamic conditions together with recognition-mediated irreversible reaction revealed that the small difference in the strength of association constants cannot be amplified beyond the limit imposed by kinetic selection.

In order to probe the origins of the limited selectivity in our dynamic recognition-mediated network, we constructed a kinetic model incorporating all of the reactions leading to the formation of exchange pool products (for details, see Supporting Information). Rather than individually measuring rate constants for each reaction, we decided to identify trends in the reactivity of each component from the distribution of the exchange pool in isolation. Simulation of the exchange pool in the absence of any maleimide (for details, see Supporting Information) showed a good agreement with the experimental data (Figure 4) presented already. Utilising our simulation, we were able to vary the rates of exchange reactions with respect to the rate of cycloaddition, setting them to be much higher, very similar or significantly lower than the rate of cycloaddition reaction. Comparison of the simulated DCL distribution to the experimentally determined results revealed that our system behaves akin to a fast exchange simulation (for details, see Supporting Information). The rates of exchange reactions have a specific effect on the selectivity for recognition products, as well as on the ratio of cis-AZ/cis-BZ within the library. Any imbalance in the concentrations of $\mathbf{A Z}$ and $\mathbf{B Z}$ generated through the irreversible reactions is erased by exchange with other library members containing the recognition and reactive materials ( $\mathbf{A}, \mathbf{B}$ and $\mathbf{Z}$ ), thus allowing the recognition-mediated reaction to proceed efficiently. In our library, the starting components are present at $20 \mathrm{mM}$, a concentration considerably above the $K_{d}$ for both competing association processes. The fast exchange reactions allow a significant concentration of both nitrones to build up, enabling both recognition processes to operate efficiently. In this respect, the dynamic scenario becomes similar to a simple kinetic experiment. We envisaged that operating the system at concentrations closer to or below the $K_{d}$ for the formation of both binary complexes, $[\mathbf{A Z} \cdot \mathbf{M}]$ and $[\mathbf{B Z} \cdot \mathbf{M}]$, would enable the initial imbalance towards one recognition product to be propagated. In this respect, we set out to investigate what conditions promote selectivity in a reaction network embedded in a DCL and whether the same conditions have a comparable influence on the network under simple kinetic selection. In order to do so, we examined the effect of varying three variables: (i) initial concentration, (ii) ratio of association constants for the formation of $[\mathbf{A Z} \cdot \mathbf{M}]$ and $[\mathbf{B Z} \cdot \mathbf{M}]$ and (iii) ratio of effective molarities for reactions of $\mathbf{A Z}$ and $\mathbf{B Z}$ nitrones. These three variables were examined under four different simulation conditions, I to IV (Figure 9). 


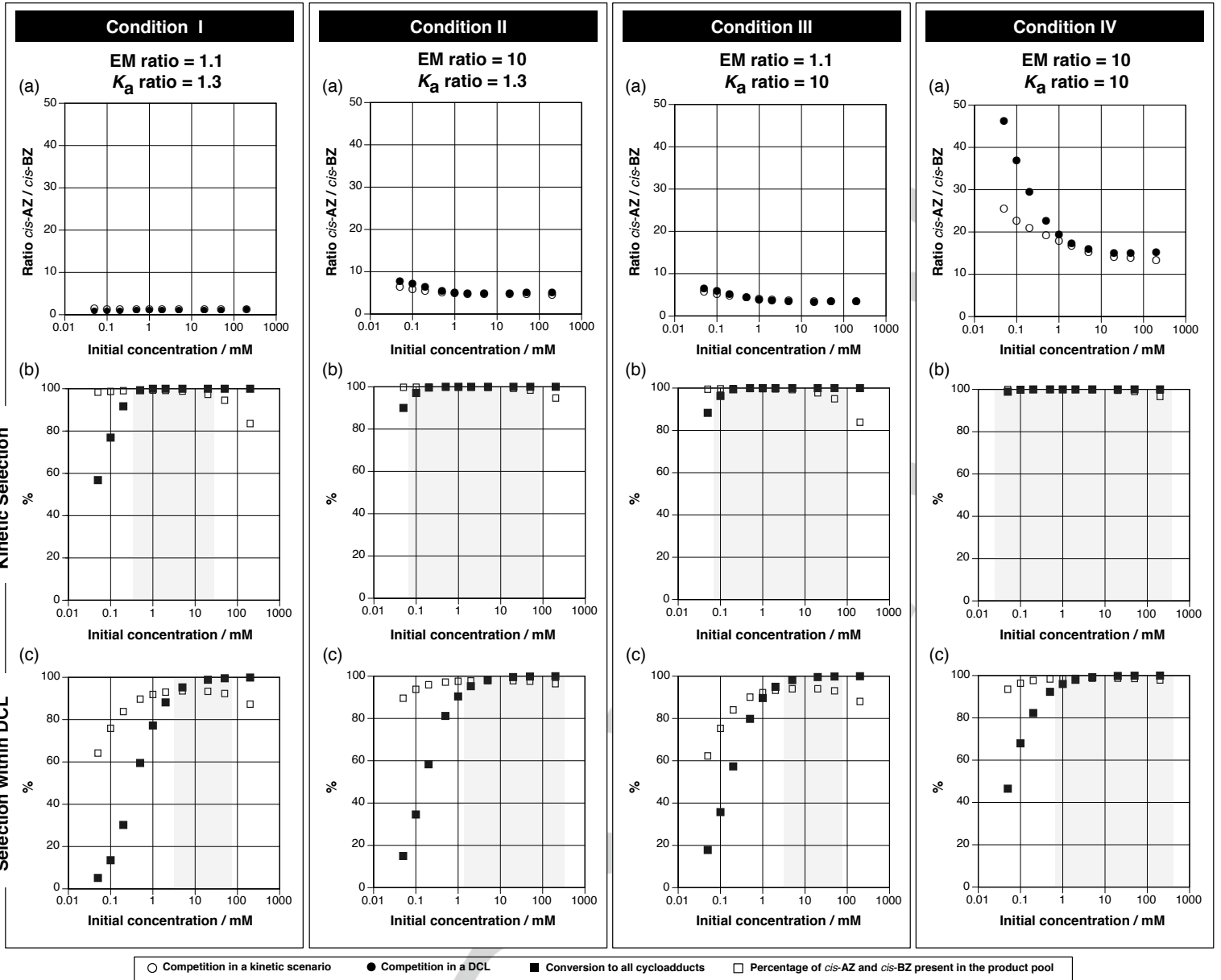

Figure 9. Outcome of simulations examining the effect of initial concentration on (a) ratio of cis-AZ/cis-BZ, (b) and (c) conversion to all cycloadducts and percentage of the recognition-enabled products in the product pool, in reaction network under kinetic selection and within a dynamic environment after 5 days, respectively. Four sets of conditions with $\mathbf{A Z / B Z}$ processes at different $K_{\mathrm{a}}$ and EM ratios were examined: Condition I employed the $K_{\mathrm{a}}$ and EM ratio corresponding to the fitted parameters determined for our experimental system, Conditions II to IV simulated either a higher ratio of EM (II), $K_{\mathrm{a}}$ (III) or both EM and $K_{\mathrm{a}}$ values (IV). Simulations were performed using the SimFit software package (Günter von Kiedrowski, University of Bochum, 2008). In each condition, grey rectangles represent the concentration range that affords the best selectivity for overall formation of cycloadducts and the highest percentage of recognitionenabled species in the cycloadduct pool (N.B.: In all cases, the x-axis is represented in logarithmic scale).

Each simulation examined a different ratio of $K_{\mathrm{a}}$ values and EM ratios at 10 different initial concentrations, ranging from 0.05 to $200 \mathrm{mM}$. We focused on examining the effect of changing initial conditions and reaction parameters on the ratio of cis-AZ/cis-BZ formed, the overall conversion to cycloadducts and percentage of recognition-enabled products (cis-AZ and cis-BZ) present in the product pool. Condition I employed the $K_{a}$ and EM ratio corresponding to the fitted parameters determined for our experimental system. Conditions II to IV simulated either a higher ratio of EM (II), $K_{\mathrm{a}}$ (III) or both EM and $K_{\mathrm{a}}$ values (IV) for the AZ/BZ recognition processes. These simulations allowed us to elucidate the effect of varying kinetic parameters at different concentrations on the ratio of cis-AZ/cis-BZ (Figure 9a) and conversion to cycloadducts after a specific time (5 days) in a kinetic scenario (Figure 9b) and in the environment of a DCL (Figure 9c).
Condition I enabled us to determine what influence different initial concentration would have on our experimental system. At $20 \mathrm{mM}$ initial concentration (Condition I), we found the simulated ratio of cis-AZ/cis-BZ within a DCL to be very similar to the experimentally determined values for dynamic selection, confirming it to be an accurate model of our dynamic library system. The simulated ratio of cis-AZ/cis-BZ is lower in the DCL selection than in the kinetic selection, and continues to fall with decreasing concentration of starting materials (for details, see Supporting Information). This observation, while counterintuitive, can be attributed to the recognition nitrone $\mathbf{A Z}$ being present at lower concentrations than the nitrone $\mathbf{B Z}$ in the library. As mentioned previously, the higher electron density around the pyridine ring in $\mathbf{B Z}$ nitrone makes the aldehyde component $\mathbf{B}$ less reactive towards hydroxylamine $\mathbf{Z}$. This effect becomes more pronounced at lower initial concentrations (for details, see Supporting Information), effectively eroding the advantage 
available to $\mathbf{A Z}$ nitrone as a result of its higher $K_{\mathrm{a}}$ value. A similar effect on the cis-AZ/cis-BZ ratio was not observed in conditions II to IV, likely because the increasing $K_{\mathrm{a}}$ and EM ratios compensate for the lower reactivity. Increasing either the $K_{\mathrm{a}}$ or EM ratio (Condition II and III) revealed that it is possible for a lower concentration to have the desired effect on the selectivity within a system, if the $K_{a}$ or EM ratios for the two competing processes are larger than those expressed by $\mathbf{A Z}$ and $\mathbf{B Z}$ in our experimental system. In fact, analysis of Condition IV revealed that a concurrent increase in both $K_{\mathrm{a}}$ and EM ratio affords an even more significant increase in selectivity for one recognition cycloadduct in the system. Nevertheless, as observed experimentally for nitrone $\mathbf{A} \mathbf{Z}$, we know from experience that increasing the strength of recognition on element $\mathbf{A}$ can potentially also affect the reactivity of this component towards nucleophiles and its ability to exchange within the library. However, as we have no means of predicting the extent to which the aldehyde reactivity might be affected, we cannot account for it in the simulation and the rates of exchange reactions are kept constant throughout the simulations. We further examined the influence of simulated initial conditions on the overall conversion to cycloadducts and the percentage of recognition-enabled species in the product pool. The simulations showed that there is an optimum window of concentration for obtaining the best selectivity for recognition products within each set of conditions. Furthermore, we found that selectivity for products formed through recognitionmediated reactions is affected more strongly by decreasing concentration within a DCL as compared to under kinetic selection. A drop in selectivity for recognition species is also observed at higher concentrations as a result of increased contribution of the bimolecular pathway to the overall product pool. However, this effect is more noticeable in conditions employing the rather low experimental EM ratio.

These kinetic simulations demonstrate that a clear set of rules exists that describes library performance. A substantial increase in selectivity for cis-AZ over cis-BZ can only be achieved under Condition IV. In this situation, both the ratio of association constants and the ratio of effective molarities for the two recognition-mediated processes within the reaction network embedded are 10. In these circumstances, better selectivity is achieved when the selection occurs from the DCL. However, the optimum concentration ranges for selectivity (grey zones, Figure 9) are significantly narrower when selection occurs from the DCL as opposed to purely kinetically. The narrowing of the optimum concentration window is proportional to the number of compounds present within the DCL - the larger the library, the narrower the window will be.

Ultimately, while the simulations here allow us to determine the effect of different recognition and reaction parameters on the selectivity generated in this system by two reactive binary complexes within a DCL, non-linear reaction processes, such as autocatalysis, are an obvious extension and implementing these processes within DCLs may, in principle, generate more dramatic selectivity for a single product, this expectation awaits experimental testing.

\section{Conclusions}

A simple recognition event has a profound effect on the distribution of a dynamic library and can be used to amplify chemical species from within a number of interconverting components. Nevertheless, the rapid exchange reactions within the dynamic library and the experimental conditions employed enable both recognition-mediated reaction processes to operate efficiently, thereby allowing kinetic selection to prevail. Through simulations and kinetic fitting, we were able to develop an understanding of the intricate interconnected network and formulate a set of rules that govern the selectivity within the dynamic system and how it compares to selectivity in a purely kinetic scenario. Finally, we have demonstrated that working at concentrations below the $K_{d}$ values of the recognition processes can positively influence the difference in selectivity between two amplified species. However, in order to induce significant selectivity in a recognition-mediated system, the difference in the association constants and the effective molarities of the employed recognition and reactive elements must be much greater than in our current experimental system. The results presented here suggest that the successful resolution of DCLs, where multiple recognition-mediated pathways are open to the system, may be impractical. Therefore, resolution of DCLs using irreversible recognition-mediated approaches may have to rely on the interplay of multiple kinetic selection modes, e.g. binary reactive complexes and autocatalytic templates, in order to be effective. This strategy is currently under investigation in our laboratory.

\section{Acknowledgements}

This work was supported by the award of a Postgraduate Studentship from EPSRC (EP/K503162/1) to TK. The research data supporting this publication can be accessed at http://dx.doi.org/10.17630/2a427a7e-d025-492e-9301$377 \mathrm{e} 18459060$. 
Keywords: molecular recognition $\cdot$ combinatorial chemistry $•$ cycloaddition reaction $\cdot$ NMR spectroscopy $\bullet$ kinetics

[1] a) J.-M. Lehn, Angew. Chemie Int. Ed. 2015, 54, 2; b) J.-M. Lehn Angew. Chem. Int. Ed. 2013, 52, 2836; c) R. J. Sarma, J. R. Nitschke, Angew. Chem. Int. Ed. 2008, 47, 377; d) V. Campbell, J. Nitschke, Synlett 2008, 20, 3077; e) T. Vicsek, Nature 2002, 418, 131; f) S. H. Strogatz, Nature 2001, 410, 268; g) G. M. Whitesides, R. F. Ismagilov, Science 1999, 284, 89; h) I. R. Epstein, K. Showalter, J. Phys. Chem. 1996, 100, 13132

[2] a) A. G. Salles, S. Zarra, R. M. Turner, J. R. Nitschke, J. Am. Chem. Soc. 2013, 135, 19143; b) N. Giuseppone, Acc. Chem. Res. 2012, 45, 2178; c) A. Vidonne, D. Philp, European J. Org. Chem. 2009, 593; d) P. T. Corbett, J. K. M. Sanders, S. Otto, Angew. Chem. Int. Ed. 2007, 46, 8858; e) R. Eelkema, M. M. Pollard, J. Vicario, N. Katsonis, B. S. Ramon, C. W. M. Bastiaansen, D. J. Broer, B. L. Feringa, Nature 2006, 440, 163; f) G. Ashkenasy, R. Jagasia, M. Yadav, M. R. Ghadiri, Proc. Natl. Acad. Sci. U. S. A. 2004, 101, 10872.

[3] a) K. Ruiz-Mirazo, C. Briones, A. de la Escosura, Chem. Rev. 2014, 114, 285; b) J. J. Peyralans, S. Otto, Curr. Opin. Chem. Biol. 2009, 13 705; c) J. R. Nitschke, Nature 2009, 462, 736; d) R. F. Ludlow, S. Otto, Chem. Soc. Rev. 2008, 37, 101; e) Stankiewicz, L. H. Eckardt, Angew. Chem. Int. Ed. 2006, 45, 342; f) M. Kindermann, I. Stahl, M. Reimold, W. M. Pankau, G. von Kiedrowski, Angew. Chem. Int. Ed. 2005, 44, 6750 .

[4] a) A. Herrmann, Chem. Soc. Rev., 2014, 43, 1899; b) Y. Jin, C. Yu, R. J. Denman, W. Zhang, Chem. Soc. Rev. 2013, 42, 6634; c) E. Moulin, G. Cormos, N. Giuseppone, Chem. Soc. Rev. 2012, 41, 1031; d) M. E. Belowich, J. F. Stoddart, Chem. Soc. Rev. 2012, 41, 2003; e) P. T. Corbett, J. Leclaire, L. Vial, K. R. West, J.-L. Wietor, J. K. M. Sanders, S. Otto, Chem. Rev. 2006, 106, 3652; f) S. J. Rowan, S. J. Cantrill, G. R. L. Cousins, J. K. M. Sanders, F. J. Stoddart, Angew. Chem. Int. Ed. 2002, 41, 898.

[5] a) J. Li, P. Nowak, S. Otto, J. Am. Chem. Soc. 2013, 135, 9222; b) S. Hamieh, V. Saggiomo, P. Nowak, E. Mattia, R. F. Ludlow, S. Otto, Angew. Chem. Int. Ed. 2013, 52, 12368; c) R. A. R. Hunt, S. Otto, Chem. Commun. 2011, 47, 847; d) P. Besenius, P. A. G. Cormack, R. F. Ludlow, S. Otto, D. C. Sherrington, Org. Biomol. Chem. 2010, 8,
2414; e) S. M. Turega, C. Lorenz, J. W. Sadownik and D. Philp, Chem. Commun., 2008, 4076; f) S. Xu and N. Giuseppone, J. Am. Chem. Soc., 2008, 130, 1826; g) P. Besenius, P. A. G. Cormack, J. Liu, S. Otto, J. K. M. Sanders and D. C. Sherrington, Chem. Eur. J., 2008, 14, 9006; h) A. Bugaut, K. Jantos, J.-L. Wietor, R. Rodriguez, J. K. M. Sanders and S. Balasubramanian, Angew. Chem. Int. Ed. 2008, 47, 2677; i) M. Chung, C. M. Hebling, J. W. Jorgenson, K. Severin, S. J. Lee, M. R. Gagne, J. Am. Chem. Soc. 2008, 130, 11819; j) P. C. Gareiss, K. Sobczak, B. R. Mcnaughton, P. B. Palde, C. A. Thornton, B. L. Miller, J. Am. Chem. Soc. 2008, 130, 16254; k) O. Sijbren, S. Kay, Top. Curr. Chem. 2007, 277, 267; I) R. M. Bennes, D. Philp, Org. Lett. 2006, 8, 3651; m) B. Brisig, J. K. M. Sanders, S. Otto, Angew. Chem. Int. Ed. 2003, 42, 1270; n) O. Ramström, J.-M. Lehn, Nat. Rev. Drug Discov. 2002, 1, 26; o) R. L. E. Furlan, G. R. L. Cousins, J. K. M. Sanders, Chem. Commun. 2000, 1761.

[6] a) Y. Zhang, O. Ramström, Chem. Eur. J. 2014, 20, 3288; b) L. Hu, O. Ramström, Chem. Commun. 2014, 50, 3792; c) Y. Zhang, L. Hu, O. Ramström, Chem. Commun. 2013, 49, 1805; d) M. Sakulsombat, Y. Zhang, O. Ramström, Top. Curr. Chem. 2012, 322, 55; e) V. del Amo, D. Philp, Chem. Eur. J. 2010, 16, 13304; f) P. Vongvilai, O. Ramström, J. Am. Chem. Soc. 2009, 131, 14419; g) M. Angelin, P. Vongvilai, A. Fischer, O. Ramström, Chem. Commun. 2008, 46, 768; h) P. Vongvilai, R. Larsson, O. Ramström, Adv. Synth. Catal. 2008, 350, 448; i) P. Vongvilai, M. Angelin, R. Larsson, O. Ramström, Angew. Chem. Int. Ed. 2007, 46, 948; j) J. D. Cheeseman, A. D. Corbett, R. Shu, J. Croteau, J. L. Gleason, R. J. Kazlauskas, J. Am. Chem. Soc. 2002, 124, 5692.

[7] a) J. M. A. Carnall, C. A. Waudby, A. M. Belenguer, M. C. A. Stuart, J. J.-P. Peyralans, S. Otto, Science 2010, 327, 1502. b) M. Barboiu, F. Dumitru, Y.-M. Legrand, E. Petit, A. van der Lee, Chem. Commun. 2009, 2192; c) M. Angelin, A. Fischer, O. Ramström, J. Org. Chem. 2008, 73, 3593; d) W. L. Noorduin, T. Izumi, A. Millemaggi, M. Leeman, H. Meekes, W. J. P. Van Enckevort, R. M. Kellogg, B. Kaptein, E. Vlieg, D. G. Blackmond, J. Am. Chem. Soc. 2008, 130, 1158.

[8] a) J. W. Sadownik, D. Philp, Angew. Chem. Int. Ed. 2008, 47, 9965; b) J. W. Sadownik, D. Philp, Org. Biomol. Chem. 2015, DOI: 10.1039/c5ob01621e. 


\section{Entry for the Table of Contents}

\section{FULL PAPER}

Two recognition-mediated processes operating through a reactive binary complex are employed for resolution of a dynamic covalent library, assembled from individual aldehydes and nucleophiles.

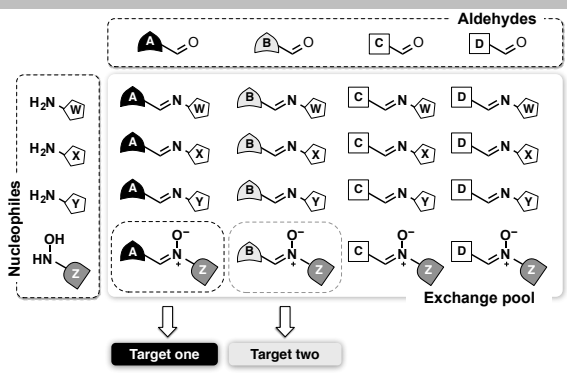

T. Kosikova, H. Mackenzie, D. Philp*

Page No. - Page No.

Probing the limits of selectivity in a recognition-mediated reaction network embedded within a dynamic covalent library 